\title{
Photocorrosion of Particles in Aqueous Solutions in an Open Cell in the Environmental Transmission Electron Microscope
}

\author{
Barnaby D.A Levin ${ }^{1}$, Diane Haiber ${ }^{1}$, Qianlang Liu ${ }^{1}$ and Peter A. Crozier ${ }^{1}$ \\ 1. School for Engineering of Matter, Transport, and Energy, Arizona State University, AZ.
}

Photocorrosion has a very large negative impact on many areas of societal importance including energy conversion technologies and structural materials. The process takes place in aqueous environments and, for semiconductors, is driven by electron-hole pair generation resulting from photon illumination [1-2]. For example, the performance of photocatalysts based on cadmium sulfide (CdS), undergoes severe degradation due to photocorrosion during photocatalytic water splitting [3]. There are many fundamental questions about the initiation and evolution of the corrosion process and how it is impacted by factors such as photon energy, temperature and the presence of solute ions. Understanding the chemical and structural changes induced by photocorrosion at the nano- and atomic scale may offer new insights into how to mitigate the process. To facilitate such studies, liquid cell holders have been developed to investigate processes taking place under aqueous conditions [4-5]. Using an environmental transmission electron microscope (ETEM), we have developed a novel open cell method for condensing liquid water around catalytic nanoparticles and illuminating the sample inside the microscope. We apply this approach to $\mathrm{CdS}$ nanoparticles in a variety of solutes and light illumination conditions to investigate photocorrosion in nanoparticles.

Samples are loaded into the ETEM in a cryogenic sample holder, which is held at approximately $5^{\circ} \mathrm{C}$ by applying a gentle heating current to the sample whilst a small quantity of liquid nitrogen is maintained in the nitrogen dewar. Our samples are then exposed to 4-10 Torr of water vapor in the ETEM column. To facilitate the formation of liquid water, CdS particles are dispersed on a TEM sample grid alongside ball milled sodium chloride ( $\mathrm{NaCl}$ ) particles (Figure 1a). As was first observed in TEM by Wise et. al. [6], $\mathrm{NaCl}$ particles will deliquesce below the saturation vapor pressure of water resulting in the formation of a salt solution around the $\mathrm{CdS}$ nanoparticles. In our experiment, liquid water droplets that form around $\mathrm{NaCl}$ particles, forming an $\mathrm{NaCl}$ solution, the concentration of which is controlled by the original mass of salt and the diameter of the liquid water droplet. The droplets envelope neighboring clusters of $\mathrm{CdS}$ particles (Figure 1b-c). Interestingly, CdS clusters are not observed to move significantly within the water droplet over time. Given that all of the particle clusters are observed to be within the bounds of the water droplet, this suggests that either the clusters are held in position either on the inside surface of the droplet, or by interaction with the carbon film at the base of the droplet. Further investigation will allow us to determine which mechanism is responsible for maintaining static CdS clusters. We are able to detect CdS and estimate the local concentration of the $\mathrm{NaCl}$ solution using in-situ X-ray energy dispersive spectroscopy (Figure 2).

We aim to study the photocorrosion of these particles by using an in-situ light system to expose the CdS particles to light whilst they are immersed in solution in the ETEM. We have modified an earlier in situ light system [7] to use a $405 \mathrm{~nm}$ laser connected to the TEM using an optical fiber inserted through the objective aperture port of the microscope to deliver a high light intensity above the bandgap of the photocatalyst sample. Aberration corrected TEM imaging before and after exposure to light and water will allow us to characterize changes that occur in clusters of particles in the same location on the TEM sample grid. Electron beam damage due to the ionization of water may be mitigated by varying, the partial 
pressure of water in the ETEM to evaporate and then re-condense liquid water to allow high resolution images to be captured from dry particles [8].

\section{References:}

[1] Gerischer, H. J. Electroanalytical Chemistry \& Interfacial Electrochemistry 82 (1977), p.133.

[2] Chen, S.Y. \& Wang, L.W. Chem. Mater. 24 (2012), p. 3659.

[3] Meissner, D. et al, Chem. Phys. Lett. 127 (1986), p. 419.

[4] Williamson, M.J. et al, Nature Materials 2 (2003), p. 532.

[5] Ross, F. M. Science. 350 (2015), aaa9886.

[6] Wise, M.E. et al, Aerosol Science and Technology 42 (2008), p. 281.

[7] Liu, Q. et al, Microsc. Microanal. 22(S3) (2016), p. 730.

[8] We gratefully acknowledge support from the U.S. DOE (DE-SC0004954) and ASU's John M. Cowley Center for High Resolution Electron Microscopy.
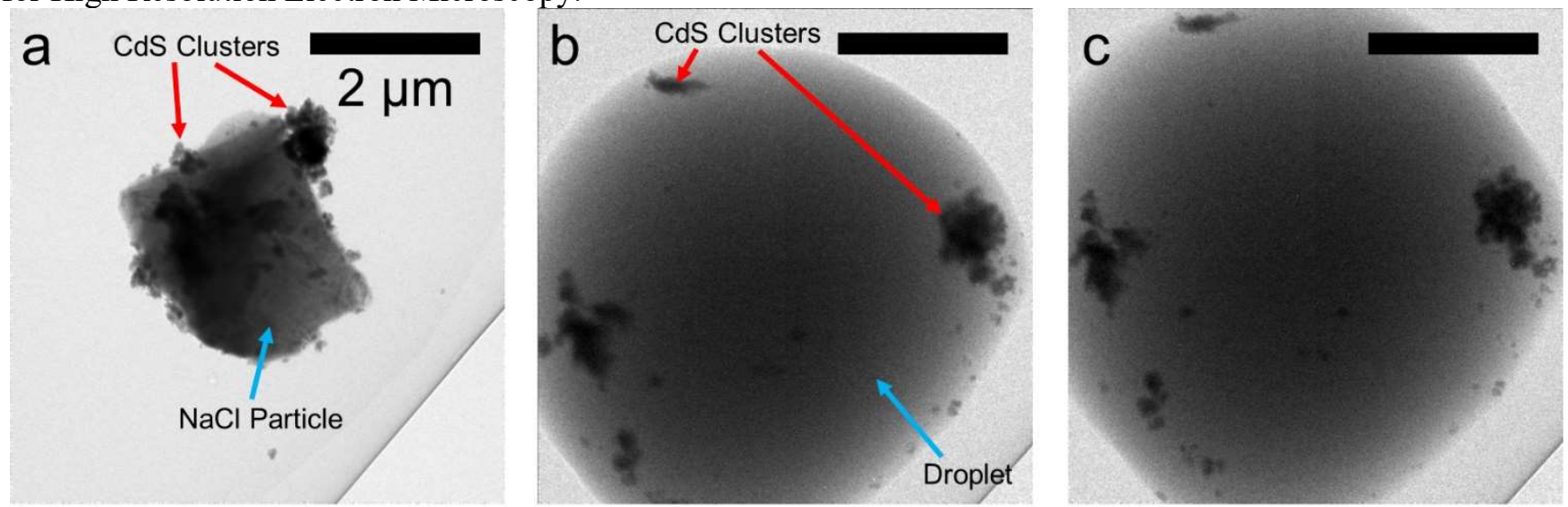

Figure 1. (a) TEM image of a typical cluster of sample particles before exposure to water vapor. (b) Image of the same area after $\sim 20$ minutes exposure to $>5$ Torr water vapor at $\sim 5^{\circ} \mathrm{C}$, showing $\mathrm{CdS}$ clusters enveloped by a water droplet. The $\mathrm{NaCl}$ appears to have dissolved in the water, forming a solution. (c) TEM image of the same area after a further 10 minutes of exposure to water vapor at $\sim 8$ Torr. The droplet of solution has increased in volume, but the relative positions of the CdS clusters appear unchanged. All scale bars are $2 \mu \mathrm{m}$.
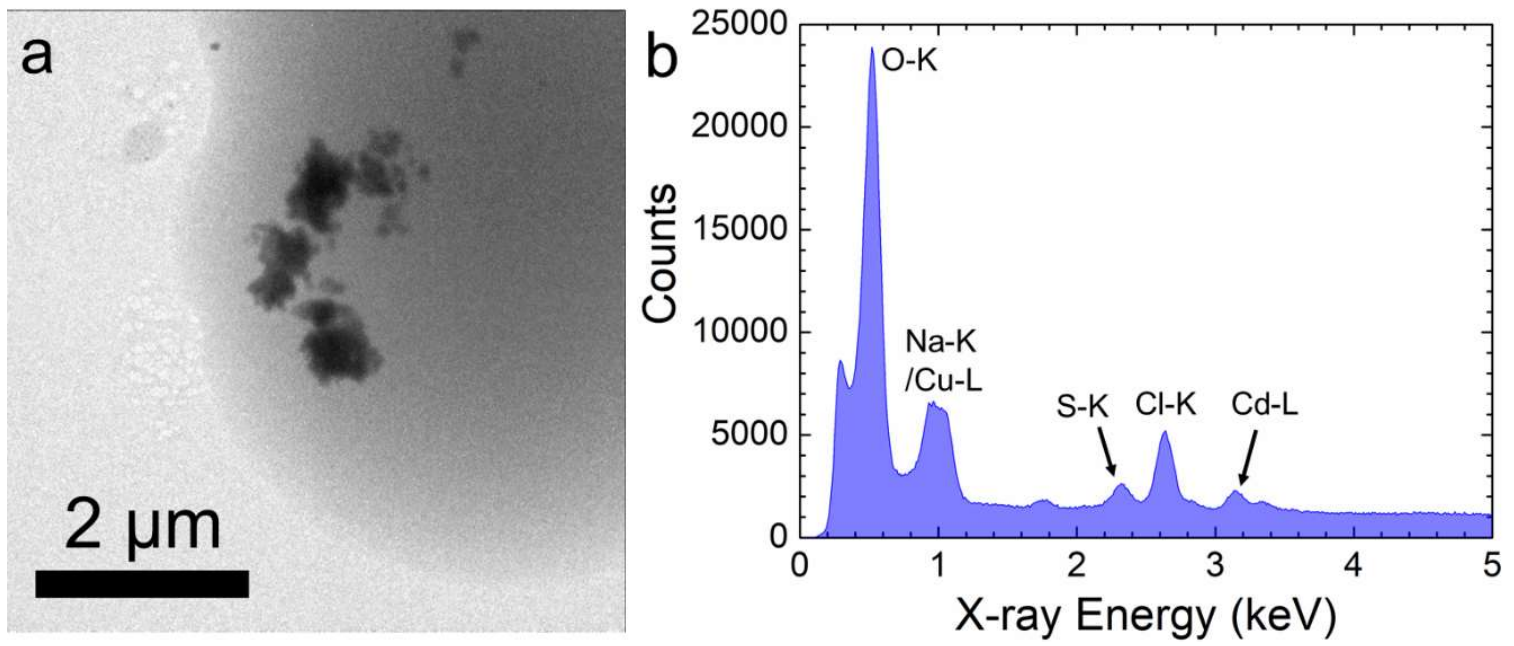

Figure 2. (a) TEM image of particles immersed in solution. (b) In situ X-ray spectrum from the field of view shown in (a). The presence of the sulfur $\mathrm{K}$ and cadmium $\mathrm{L}$ edges confirms that we are able to detect $\mathrm{CdS}$ particles spectroscopically when they are enveloped in liquid. Comparison of the relative the size of the $\mathrm{Cl}-\mathrm{K}$ and $\mathrm{O}-\mathrm{K}$ peaks with spectra simulated using NIST DTSA II suggests that the $\mathrm{NaCl}$ solution has a concentration of $\sim 0.03 \mathrm{M}$ in this area. 